\title{
GMR
}

\section{Influence of ERCC2 gene polymorphisms on the treatment outcome of osteosarcoma}

\author{
Z.F. Liu, A.L.J. Asila, K. Aikenmu, J. Zhao, Q.C. Meng and R. Fang \\ Department of Orthopaedics, \\ Chinese Medicine Hospital of Xinjiang Uygur Autonomous Region, Urumchi, China \\ Corresponding author: R. Fang \\ E-mail: ruifangr@163.com \\ Genet. Mol. Res. 14 (4): 12967-12972 (2015) \\ Received March 30, 2015 \\ Accepted June 25, 2015 \\ Published October 21, 2015 \\ DOI http://dx.doi.org/10.4238/2015.October.21.17
}

ABSTRACT. We conducted a prospective study to investigate the role of ERCC2 gene polymorphisms on the outcome of cisplatinbased treatment in patients with osteosarcoma. A total of 115 patients with osteosarcoma were included in our study. Genotyping of ERCC2 Asn312Asp (rs1799793) and Lys751Gln (rs13181) was performed using a matrix-assisted laser desorption/ionization time-of-flight mass spectrometry method. Of the 115 patients, 78 showed complete or partial response to chemotherapy, with a response rate of $67.85 \%$. Our study suggested that the AA genotype of ERCC2 Asn312Asp was associated with a better response to chemotherapy, and the related adjusted OR $(95 \% \mathrm{Cl})$ was 4.85 (1.06-42.71). By Cox proportional hazards model analysis, we found that the AA genotype of ERCC2 Asn312Asp was associated with longer overall survival of patients with osteosarcoma when compared with the $\mathrm{GG}$ genotype, and the hazards ratio $(95 \% \mathrm{Cl})$ for the AA genotype was 0.65 (0.27-1.47). In conclusion, our study found that the ERCC2 Asn312Asp gene polymorphism likely plays an important role in influencing the chemotherapy response and overall survival of patients with osteosarcoma.

Key words: ERCC2; Polymorphism; Osteosarcoma; Treatment outcome 


\section{INTRODUCTION}

Osteosarcoma is derived from mesenchymal tissues, and often occurs in the distal femur, proximal tibia, and humeral metaphysis. Osteosarcoma is frequently diagnosed in children and adolescents (Mirabello et al., 2009; Ottaviani and Jaffe, 2009). Although advances have been made in surgery and chemotherapy treatments for osteosarcoma, the rates of survival of this cancer are not satisfactory (Chou and Gorlick, 2006). Most treatments for osteosarcoma are based on multiagent preoperative and postoperative chemotherapy, including cisplatin in combination with doxorubicin and high-dose methotrexate (Quartuccio et al., 2013). Although these active chemotherapeutic agents have been available for many years, inter-individual efficacy and toxicities are still prevalent among patients with similar disease, which led us to hypothesize that single nucleotide polymorphisms (SNPs) in drug metabolism enzymes might play a role in ultimate drug exposure and thus affect the chemotherapeutic outcomes and toxicities.

Cisplatin binds to DNA and forms DNA adducts including both intrastrand and interstrand crosslinks, and inhibits DNA replication (Marsh et al., 2009). Changes in DNA induced by exposure to environmental and endogenous agents such as cisplatin might result in genetic instability and mutagenesis. Genetic variations in DNA repair genes can change the amount or activity of the encoded proteins, and therefore might determine individual response to cisplatin. ERCC2 is an important DNA repair enzyme and is responsible for resistance to cisplatin. In our study, we conducted a prospective study to investigate the role of ERCC2 gene polymorphisms on the outcome of cisplatin-based treatment in patients with osteosarcoma.

\section{MATERIAL AND METHODS}

\section{Patients}

This study included 115 histologically confirmed patients with osteosarcoma. We included all patients with osteosarcoma that were treated with cisplatin-based chemotherapy from the Department of Orthopaedics, the Chinese Medicine Hospital of Xinjiang Uygur Autonomous Region. All subjects gave their informed consent prior to inclusion in the study. The protocol of this study was approved by the Ethics Committee for Research in Medicine according to the Declaration of Helsinki.

\section{Evaluation of treatment outcome}

Tumor responses were evaluated by contrasted computed tomography scans and/ or magnetic resonance imaging every two cycles to document complete response (CR), partial response $(\mathrm{PR})$, stable disease, or progressive disease according to the Response Evaluation Criteria in Solid Tumors (RECIST criteria) version 1.1 (Eisenhauer et al., 2009). Overall survival was calculated from the start of chemotherapy to the date of death from all causes or at the end of follow-up.

\section{DNA extraction and genotyping}

Each patient was asked to provide a 5-mL blood sample. Blood samples were collected in ethylenediaminetetraacetic acid-containing tubes, and the sera were separated and stored at $-70^{\circ} \mathrm{C}$ for future use. Genomic DNA was isolated from the blood samples of the patients with 
osteosarcoma using a Qiagen genomic DNA isolation kit (Venlo, The Netherlands) according to manufacturer instructions. Genotyping of ERCC2 Asn312Asp (rs1799793) and Lys751GIn (rs13181) was performed with a matrix-assisted laser desorption/ionization time-of-flight mass spectrometry method (Sequenom, Inc., San Diego, CA, USA). DNA was amplified by polymerase chain reaction (PCR) with primers designed with Assay Designer 4.0 (Sequenom). To verify the results, $5 \%$ of the DNA samples were randomly selected for duplicate assays. All the results were generated and analyzed by laboratory staff unaware of patient status.

\section{Statistical analysis}

The SPSS software version 17.0 (SPSS, Chicago, IL, USA) was used to conduct the analyses. The associations between genotype polymorphisms of ERCC2 Asn312Asp (rs1799793) and Lys751Gln (rs13181) and response to chemotherapy in patients with osteosarcoma were further estimated by $\mathrm{OR}$ and $95 \% \mathrm{Cl}$ in a logistic regression model. The Kaplan-Meier method was used to calculate progression free survival and a log-rank test was used to compare the overall survival differences among the different genotypes. The hazards ratio $(\mathrm{HR})$ and $95 \% \mathrm{Cl}$ of significant genotypes for the overall survival of patients with osteosarcoma were estimated by a Cox hazards proportional model with adjustments for potential confounding factors. All $\mathrm{P}$ values were based on two-tailed tests and $\mathrm{P}<0.05$ was set as the level of significance.

\section{RESULTS}

The demographic and clinical characteristics of patients with osteosarcoma are shown in Table 1. Of 115 patients, $65(56.52 \%)$ patients were males and $50(43.48 \%)$ were females; $71(61.74 \%)$ were below 20 years old; 90 (78.26\%) were at tumor stage I-II; $35(30.43 \%)$ were diagnosed with osteoblastic osteosarcoma, $53(46.09 \%)$ with chondroblastic osteosarcoma, and tumor locations of $81(70.43 \%)$ were at the extremities.

\begin{tabular}{|c|c|c|}
\hline Characteristics & Patients $(\mathrm{N})$ & $\%$ \\
\hline \multicolumn{3}{|l|}{ Gender } \\
\hline Male & 65 & 56.52 \\
\hline Female & 50 & 43.48 \\
\hline \multicolumn{3}{|l|}{ Age } \\
\hline$<20$ & 71 & 61.74 \\
\hline$\geq 20$ & 44 & 38.26 \\
\hline \multicolumn{3}{|l|}{ Tumor stage } \\
\hline $\mathrm{I}-\mathrm{II}$ & 90 & 78.26 \\
\hline III-IV & 25 & 21.74 \\
\hline \multicolumn{3}{|l|}{ Histological type } \\
\hline Osteoblastic & 35 & 30.43 \\
\hline Chondroblastic & 53 & 46.09 \\
\hline Fibroblastic & 12 & 10.43 \\
\hline Other & 15 & 13.04 \\
\hline \multicolumn{3}{|l|}{ Tumor location } \\
\hline Extremities & 81 & 70.43 \\
\hline Other & 34 & 29.57 \\
\hline \multicolumn{3}{|c|}{ Response to chemotherapy } \\
\hline $\mathrm{CR}+\mathrm{PR}$ & 78 & 67.83 \\
\hline$S D+P D$ & 37 & 32.17 \\
\hline
\end{tabular}


The associations between variants of ERCC2 Asn312Asp and ERCC2 Lys751GIn and chemotherapy response are shown in Table 2. Of the 115 patients, 78 showed $C R$ and PR to chemotherapy, with a response rate of $67.85 \%$. Our study suggested that the AA genotype of ERCC2 Asn312Asp was associated with a better response to chemotherapy; the related adjusted OR $(95 \% \mathrm{Cl})$ was $4.85(1.06-42.71)$. However, we did not find significant association between genetic variation of ERCC2 Lys751GIn and response to chemotherapy.

\begin{tabular}{|c|c|c|c|c|c|c|}
\hline Genotypes & $C R+P R$ & $\%$ & $S D+P D$ & $\%$ & OR $(95 \% \mathrm{Cl})^{1}$ & $P$ value \\
\hline \multicolumn{7}{|c|}{ ERCC2 Asn312Asp } \\
\hline GG & 32 & 41.03 & 22 & 59.46 & 1.0 (Ref.) & \\
\hline GA & 34 & 43.59 & 13 & 35.14 & $1.96(0.78-4.86)$ & 0.14 \\
\hline AA & 12 & 15.38 & 2 & 5.41 & $4.85(1.06-42.71)$ & 0.03 \\
\hline $\mathrm{GA}+\mathrm{AA}$ & 46 & 58.97 & 15 & 40.54 & $2.30(0.92-5.18)$ & 0.06 \\
\hline \multicolumn{7}{|c|}{ ERCC2 Lys751GIn } \\
\hline AA & 29 & 37.18 & 17 & 45.95 & 1.0 (Ref.) & \\
\hline$A C$ & 36 & 46.15 & 16 & 43.24 & $1.47(0.61-3.77)$ & 0.45 \\
\hline $\mathrm{CC}$ & 13 & 16.67 & 4 & 10.81 & $2.16(0.52-10.72)$ & 0.26 \\
\hline$A C+C C$ & 49 & 62.82 & 20 & 54.05 & $1.48(0.63-3.50)$ & 0.25 \\
\hline
\end{tabular}

${ }^{1}$ Adjusted for gender, age, tumor stage, location, and histological type. $C R=$ complete response; $P R=$ partial response; $\mathrm{SD}=$ stable disease $; \mathrm{PD}=$ progressive disease.

By Cox proportional hazard model analysis, we found that the AA genotype of ERCC2 Asn312Asp was associated with longer overall survival of patients with osteosarcoma when compared with the GG genotype; the HR $(95 \% \mathrm{Cl})$ for the AA genotype was $0.23(0.021-0.97)$ after adjusting for potential confounding factors (Table 3). However, we observed no significant association between the ERCC2 Lys751G $\mathrm{ln}$ gene polymorphism and the overall survival of patients with osteosarcoma.

Table 3. Association between investigated gene polymorphisms and overall survival in patients with osteosarcoma.

\begin{tabular}{|c|c|c|c|c|c|c|}
\hline Genotypes & Total & $\%$ & Event & $\%$ & $\mathrm{HR}(95 \% \mathrm{Cl})^{1}$ & $P$ value \\
\hline \multicolumn{7}{|c|}{ ERCC2 Asn312Asp } \\
\hline GG & 54 & 47.6 & 22 & 52.38 & Ref. (1.0) & - \\
\hline GA & 47 & 40.6 & 18 & 42.86 & $0.87(0.34-1.94)$ & 0.71 \\
\hline AA & 14 & 11.8 & 2 & 4.76 & $0.23(0.021-0.97)$ & 0.04 \\
\hline$G A+A A$ & 61 & 52.4 & 20 & 47.62 & $0.65(0.27-1.47)$ & 0.28 \\
\hline \multicolumn{7}{|c|}{ ERCC2 Lys751GIn } \\
\hline$A A$ & 46 & 40.2 & 17 & 40.48 & Ref. (1.0) & - \\
\hline$A C$ & 52 & 45.2 & 19 & 45.24 & $0.95(0.36-2.18)$ & 0.81 \\
\hline $\mathrm{CC}$ & 17 & 14.6 & 6 & 14.29 & $0.88(0.25-3.57)$ & 0.75 \\
\hline$A C+C C$ & 69 & 59.8 & 25 & 59.52 & $0.95(0.38-2.05)$ & 0.63 \\
\hline
\end{tabular}

${ }^{1}$ Adjusted for gender, age, tumor stage, location, and histological type. $\mathrm{HR}=$ hazards ratio.

\section{DISCUSSION}

In our study, we investigated the influence of the Asn312Asp and Lys751GIn polymorphisms in the DNA repair gene ERCC2 gene on the treatment response and overall survival of patients with osteosarcoma treated with chemotherapy. Our study found that the ERCC2 Asn312Asp gene polymorphism could influence the response to chemotherapy and the overall survival of patients with osteosarcoma. 
Our study reported that the AA genotype of ERCC2 Asn312Asp was associated with better response to chemotherapy and overall survival of patients with osteosarcoma, even after adjusting for potential confounding factors. Furthermore, ERCC2 Asn312Asp has been found to be an important genetic factor influencing the clinical outcome of several other kinds of cancers (Biason et al., 2012; Dong et al., 2012; Li et al., 2013; Qin et al., 2013; Rumiato et al., 2013; Yang and Xian, 2014; Zhou et al., 2014; Goričar et al., 2015). However, the results of these studies are inconsistent. Li et al. (2013) reported that the AA genotype of ERCC2 Asn312Asp was associated with significantly better overall survival of patients with gastric cancer, a result similar to that obtained by Zhou et al. (2014), who also demonstrated a better response to chemotherapy. Dong et al. (2012), on the other hand, did not find that ERCC2 Asn312Asp gene polymorphism was associated with overall survival of non-small cell lung carcinoma (NSCLC). Yang and Xian (2014) conducted a meta-analysis with 46 studies, and they found that ERCC2 rs1799793 might be a useful biomarker to predict the clinical outcome of patients with NSCLC treated with platinum-based chemotherapy. However, some studies did not find significant association between the ERCC2 Asn312Asp gene polymorphism and prognosis of cancer. Rumiato et al. (2013) did not find that the ERCC2 Asn312Asp gene polymorphism was a predictive marker in patients with esophageal cancer treated with cisplatin-based chemotherapy. Similarly, Qin et al. (2013) conducted a metaanalysis with 24 studies, and they found that the ERCC2 Asn312Asp polymorphism was not associated with response to chemotherapy in patients with NSCLC. These inconsistent results might be due to differences between the studies in ethnicities, sources of patients, types of tumors, sample sizes, or to chance.

For the association between the ERCC2 Asn312Asp gene polymorphism and overall survival of osteosarcoma, three previous studies have reported positive findings (Biason et al., 2012; Goričar et al., 2015; Ji and He, 2015). Biason et al. (2012) suggesting that ERCC2Asn312Asp could influence the response to chemotherapy and the overall survival of osteosarcoma. Goričar et al. (2015) found that the ERCC2 rs1799793 variant allele was associated with longer event-free survival in osteosarcoma. However, Ji and He (2015) did not found that the ERCC2 Asn312Asp gene polymorphism could influence the clinical outcome in patients with osteosarcoma. Therefore, further studies are greatly needed to confirm the association between the ERCC2 Asn312Asp gene polymorphism and the clinical outcome of osteosarcoma.

In conclusion, our study found that the ERCC2 Asn312Asp gene polymorphism plays an important role in influencing the chemotherapy response and overall survival of patients with osteosarcoma. The ERCC2 Asn312Asp polymorphism might substantially contribute to the future design of individualized cancer treatments for patients with osteosarcoma.

\section{Conflicts of interest}

The authors declare no conflict of interest.

\section{REFERENCES}

Biason P, Hattinger CM, Innocenti F, Talamini R, et al. (2012). Nucleotide excision repair gene variants and association with survival in osteosarcoma patients treated with neoadjuvant chemotherapy. Pharmacogenomics J. 12: 476-483.

Chou AJ and Gorlick R (2006). Chemotherapy resistance in osteosarcoma: current challenges and future directions. Expert Rev. Anticancer Ther. 6: 1075-1085.

Dong J, Hu Z, Shu Y, Pan S, et al. (2012). Potentially functional polymorphisms in DNA repair genes and non-small-cell lung cancer survival: a pathway-based analysis. Mol. Carcinog. 51: 546-552. 
Eisenhauer EA, Therasse P, Bogaerts J, Schwartz LH, et al. (2009). New response evaluation criteria in solid tumours: revised RECIST guideline (version 1.1). Eur. J. Cancer. 45: 228-247.

Goričar K, Kovač V, Jazbec J, Zakotnik B, et al. (2015). Genetic variability of DNA repair mechanisms and glutathione-Stransferase genes influences treatment outcome in osteosarcoma. Cancer Epidemiol. 39: 182-188.

Ji WP and He NB (2015). Investigation on the DNA repaired gene polymorphisms and response to chemotherapy and overall survival of osteosarcoma. Int. J. Clin. Exp. Pathol. 8: 894-899.

Li Y, Liu Z, Liu H, Wang LE, et al. (2013). ERCC1 and ERCC2 variants predict survival in gastric cancer patients. PLoS One 8: e71994.

Marsh S, McLeod H, Dolan E, Shukla SJ, et al. (2009). Platinum pathway. Pharmacogenet. Genomics 19: 563-564.

Mirabello L, Troisi RJ and Savage SA (2009). Osteosarcoma incidence and survival rates from 1973 to 2004: Data from the surveillance, epidemiology, and end results program. Cancer 115: 1531-1543.

Ottaviani G and Jaffe N (2009). The epidemiology of osteosarcoma. Cancer Treat. Res. 152: 3-13.

Qin Q, Zhang C, Yang X, Zhu H, et al. (2013). Polymorphisms in XPD gene could predict clinical outcome of platinum-based chemotherapy for non-small cell lung cancer patients: a meta-analysis of 24 studies. PLoS One 8: e79864.

Quartuccio N, Treglia G, Salsano M, Mattoli MV, et al. (2013). The role of fluorine-18-fluorodeoxyglucose positron emission tomography in staging and restaging of patients with osteosarcoma. Radiol. Oncol. 47: 97-102.

Rumiato E, Cavallin F, Boldrin E, Cagol M, et al. (2013). ERCC1 C8092A (rs3212986) polymorphism as a predictive marker in esophageal cancer patients treated with cisplatin/5-FU-based neoadjuvant therapy. Pharmacogenet. Genomics 23: 597-604.

Yang $Y$ and Xian $L$ (2014). The association between the ERCC1/2 polymorphisms and the clinical outcomes of the platinumbased chemotherapy in non-small cell lung cancer (NSCLC): a systematic review and meta-analysis. Tumour Biol. 35 : 2905-2921.

Zhou J, Liu ZY, Li CB, Gao S, et al. (2014). Genetic polymorphisms of DNA repair pathways influence the response to chemotherapy and overall survival of gastric cancer. Tumour Biol. 36: 3017-3023. 